\begin{tabular}{|c|c|}
\hline Title & Topology Optimization A ccelerated by Deep Learning \\
\hline Author(s) & Sasaki, Hidenori; Igarashi, Hajime \\
\hline Citation & $\begin{array}{l}\text { IEEE Transactions on Magnetics, 55(6), } 7401305 \\
\text { https://doi.org/10.1109/ MA G.2019.2901906 }\end{array}$ \\
\hline Issue Date & $2019-06$ \\
\hline Doc URL & http://hdl.handle.net/2115/74695 \\
\hline Rights & $\begin{array}{l}\text { (c) } 2019 \text { IEEE. Personal use of this material is permitted. Permission from IEEE must be obtained for all other uses, in } \\
\text { any current or future media, including reprinting/republishing this material for advertising or promotional purposes, } \\
\text { creating new collective works, for resale or redistribution to servers or lists, or reuse of any copyrighted component of } \\
\text { this work in other works. }\end{array}$ \\
\hline Tyре & article (author version) \\
\hline File Information & MAGCON-18-11-1747-final.pdf \\
\hline
\end{tabular}

Instructions for use 


\title{
Topology Optimization Accelerated by Deep Learning
}

\author{
Hidenori Sasaki ${ }^{1}$, Hajime Igarashi ${ }^{1}$, IEEE Member \\ ${ }^{1}$ Graduate School of Information Science and Technology, Hokkaido University, Sapporo, Japan
}

\begin{abstract}
The computational cost of topology optimization based on stochastic algorithm is shown to be greatly reduced by deep learning. In the learning phase, the cross-sectional image of an IPM motor, represented in RGB, is used to train a convolutional neural network (CNN) to infer the torque properties. In the optimization phase, all the individuals are approximately evaluated by the trained CNN, while finite element analysis for accurate evaluation is performed only for a limited number of individuals. It is numerically shown that computational cost for the topology optimization can be reduced without loss of optimization quality.
\end{abstract}

Index Terms - Deep learning, Convolutional neural network, Topology optimization, Approximate computing, IPM motor.

\section{INTRODUCTION}

$\mathrm{T}$ HE the parameter optimization of electric machines seeks for an optimal solution in the space spanned by the userdefined design variables such as length, nodal position, angle and so on. While this approach has been widely used in industries, it would be difficult to choose the adequate design variables to find a solution with a satisfactory performance. In contrast, the topology optimization does not require setting of the design variables. Moreover, because of the high flexibility in the topology optimization, which allows appearance and disappearance of holes in a design region and free modification of material boundaries, one can find a novel machine structure with excellent performance. It has been shown that the topology optimization based on stochastic algorithm, especially the genetic algorithm (GA), is fairly suitable for the topology optimization of rotating machines [1, 2]. However, this approach requires large computational cost because of a huge number of fitness evaluations using. e.g, the finite element method (FEM).

For reduction of the computational cost in optimization, the approximate computing based on, for example, the response surface method [3] and Kriging method [4] would be effective when the dimension of the design space is less than, say, ten. However, the result of the topology optimization is, in general, an image whose degree of freedoms is far larger than that adequate for the above methods. The artificial neural network (ANN) has been shown effective for the approximate computing for the parameter optimization [5]. It is, however, often difficult to make an adequate choice of the image features to build the input data for ANN. In contrast, when using deep learning (DL) for image processing, image can be directly input to a DL system without the feature design [6].

In this paper, we propose the use of DL for reduction of computing burden in the topology optimization. The authors have shown that DL can accurately infer the torque performance of an interior permanent magnet (IPM) motor

Manuscript received April 1, 2015; revised May 15, 2015 and June 1, 2015; accepted July 1, 2015. Date of publication July 10, 2015; date of current version July 31, 2015. (Dates will be inserted by IEEE; "published" is the date

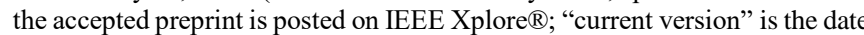
the typeset version is posted on Xplore $\left.{ }^{\circledR}\right)$. Corresponding author: H. Igarashi (e-mail: igarashi@ssi.ist.hokudai.ac.jp).

Digital Object Identifier (inserted by IEEE). from the cross-sectional image in RGB [7]. In this paper, it is shown that DL is indeed effective for the reduction of computing complexity in the topology optimization. The several ways of the possible use of DL for this purpose are also proposed.

\section{TOPOLOGY OPTIMIZATION WITH AID OF DEEP LEARNING}

\section{A. Topology Optimization Method}

We adopt the topology optimization based on the normalized Gaussian function [1,2] for the test of the proposed method, which will be briefly described below, while the proposed method can be applied to any topology optimization method in principle. For the numerical examples, we optimize the structure of an IPM motor. To evaluate the performance of an IPM motor, magnetostatic magnetic field is analyzed by FEM.

We introduce the shape function $\varphi$ defined by

$$
\begin{array}{r}
\varphi(\boldsymbol{x})=\sum_{i=1}^{n} w_{i} \tilde{G}\left(\boldsymbol{x}-\boldsymbol{x}_{\boldsymbol{i}}\right), \\
\tilde{G}\left(\boldsymbol{x}-\boldsymbol{x}_{\boldsymbol{i}}\right) \equiv \frac{G\left(\boldsymbol{x}-\boldsymbol{x}_{\boldsymbol{i}}\right)}{\sum_{j=1}^{n} G\left(\boldsymbol{x}-\boldsymbol{x}_{\boldsymbol{j}}\right)}
\end{array}
$$

where $G$ denotes the Gaussian function of the $d$-th dimension that is centered at $\boldsymbol{x}_{i}, i=1,2, \ldots n$. The Gaussians are uniformly deployed in the design region. Because we consider here twodimensional magnetic fields, $d$ is set to 2 . The material attribute $A_{e}=\{$ iron, air $\}$ of finite element $e$ is determined from

$$
\begin{aligned}
& A_{e}=\text { "iron" if } \varphi\left(\boldsymbol{x}_{e}\right) \geq 0, \\
& A_{e}=\text { "air" else, }
\end{aligned}
$$

where $\boldsymbol{x}_{e}$ is the gravitational center of element $e$. In the topology optimization, the weights $w_{i}, i=1,2, \ldots, n$, are determined, using GA in this study, so that the cost function is minimized. The standard deviation included in the Gaussian function is taken larger than the size of the finite elements to avoid convergence to complicated structures.

\section{B. Deep Learning}

We adopt DL based on the convolutional neural network (CNN) [6] to perform fast evaluation of the performance of an IPM motor. In this study, the cross-sectional images in RGB $(N=3)$ appeared during the optimization process of an IPM motor are chosen for the input data to CNN. It is also possible to use the weighting coefficients $w_{i}$ in (1a) as the input data to 
CNN. However, they are highly problem-dependent. Hence, the CNN trained with $w_{i}$ is no more valid for other models with a different design region or different $n$. On the other hand, the image has universality in a sense that many image features are common for different models. For this reason, we use the image as the input to CNN. In our study, CNN is trained to classify different-shaped motor models into categories relevant to the grade of motor properties such as average torque, torque ripple and efficiency.

\section{Use of Deep Learning for Optimization}

There are two possible ways in the use of DL for acceleration of the topology optimization: on-line and off-line methods. In the former, CNN is trained during an optimization process, whereas $\mathrm{CNN}$ is trained in the learning phase prior to the optimization in the latter case. We will make a discussion about the former elsewhere, while we confine ourselves to the latter in this paper.

The data for training of CNN has to include pairs <image, property> with sufficient diversity. When considering the average torque of an electric motor, for example, we need as uniform distribution of average torque as possible. It would be hard to obtain such data by randomly generation of input images because this would provide only low-torque motors. For this reason, we generate the training data by performing a preliminary topology optimization to maximize average torque which would provide a set of motors with well-distributed torques. Then the trained $\mathrm{CNN}$ is used for acceleration of the topology optimization.

Possible ways for the effective use of CNN in the topology optimization are listed in Table 1. In case (i), the trained CNN is used for the optimization problems $\pi_{2}, \pi_{3}$ etc. which include cost functions or constraints that are different from those in the preliminary optimization $\pi_{1}$. In case (ii), $\mathrm{CNN}$ is trained with the data obtained by the optimization for model $\mathrm{m}_{1}$. Then the trained $\mathrm{CNN}$ is used as the approximate computing method in the optimization for different models $\mathrm{m}_{2}, \mathrm{~m}_{3}$, etc. In the optimization of an IPM motor, the different model would have different magnet position or motor radius, for example. Similarly, in (iii), CNN trained for a set of random seeds in the GA process is used in the optimization phase with different sets of random seeds. In (iv), CNN is firstly trained with the data obtained by the optimization with small population size in GA. Then the trained CNN is used for the optimization with larger population size. In this paper, the feasibility of the proposed method for cases (i) and (ii) is discussed with numerical results. Case (iv) is effective especially for multi-objective problems that need a large computational cost, which is discussed in [8].

Table I Use of deep learning for optimization

\begin{tabular}{lll}
\hline \hline & Learning phase & Optimization phase \\
\hline (i) & problem $\pi_{1}$ & problems $\pi_{2}, \pi_{3}, \ldots$ \\
(ii) & model $\mathrm{m}_{1}$ & models $\mathrm{m}_{2}, \mathrm{~m}_{3}, \ldots$ \\
(iii) & random seed $\mathrm{s}_{1}$ & random seed $\mathrm{s}_{2}, \mathrm{~s}_{3}, \ldots$ \\
(iv) & small population size & large population size \\
\hline \hline
\end{tabular}

\section{Reduction in Number of Fitness Evaluation with FEM}

In the optimization phase, the fitness evaluation can be performed only using the trained $\mathrm{CNN}$ without FE analysis. However, this might result in an unsuccessful optimization result because of the error in the evaluation with CNN. For this reason, we introduce the probability $p$ of FE analysis depending on the category $F$ of an individual provided by CNN. For the individual belonging to a category with low performance, $p$ is set low because they would not survive in the next generation. In contrast, the additional FE analysis for highly accurate evaluation is made at high $p$ for individuals in categories with high performance. The design of probability distribution function $P(F)$ will be exemplified in the next section.

\section{NUMERICAL RESULTS}

\section{A. Application to different problem}

For a numerical example of (i) in Table I, we consider the topology optimization of the rotor of the IPM motor shown in Fig.1, which include 42 Gaussian bases represented by circles that have the same radius of the standard deviation and are centered at $\boldsymbol{x}_{i}, i=1,2, \ldots, 42$. The optimization settings are summarized in Table II. In the learning phase, the training data for CNN is obtained by solving the preliminary optimization problem in which the average torque $T_{\text {ave }}$ is maximized:

$$
F_{1}=\frac{T_{\text {ave }}}{T_{\text {ave }}^{0}} \rightarrow \text { max. }
$$

where the normalized constant is set as $T_{\text {ave }}^{0}=2.1 \mathrm{Nm}$, which is the average torque of the original model before optimization. Problem (3) is solved using GA, where we impose the constraint that the iron core is not separated into parts. This constraint is also imposed in the following problems. The torque ripple, $T_{\text {rip }}=\left(T_{\max }-T_{\min }\right) / T_{\mathrm{ave}}$, of the individual generated in the GA process is also evaluated by FEM and stored. By solving (3), we obtain 168,000 samples of <motor image, $\left(T_{\text {ave }}, T_{\text {rip }}\right)>$. The samples are classified into eight categories shown in Table III with reference to the values of $T_{\text {ave }}$ and $T_{\text {rip }}$ computed by FEM, and they are provided to CNN. It is found that the classification with respect to $T_{\text {rip }}$ is more difficult than that w.r.t. $T_{\text {ave }}$. Hence, we train CNN with 4,000 and 10,000 data for classification of $T_{\text {ave }}$ and $T_{\text {rip }}$, respectively, and the remained data that is not used for the training is used for the test of the trained $\mathrm{CNN}$ for cross validation.

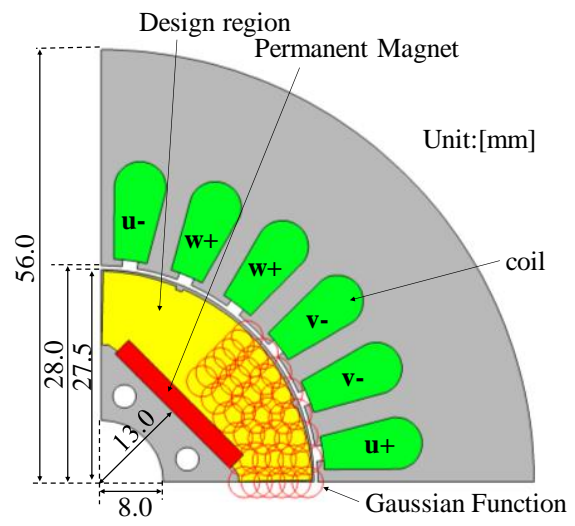

Fig.1 IPM motor

Circles represent contour of Gaussian-basis functions 
The accuracy of the trained CNN is summarized in the confusion matrix shown in Tables IV and V. It is found that samples of $99 \%$ and $81 \%$ are classified by $\mathrm{CNN}$ to the categories within the error range of \pm 0.1 compared to those classified by FEM. Since the classification by the trained CNN is not perfect, the additional FE analysis for a subset of individuals would be necessary in the optimization phase.

In the optimization phase, the different problem

$$
F_{2}=1.3 \frac{T_{\text {ave }}}{T_{\text {ave }}^{0}}-0.3 \frac{T_{\text {rip }}}{T_{\text {rip }}^{0}} \rightarrow \text { max. }
$$

is solved with aid of the trained $\mathrm{CNN}$, where $T_{\text {rip }}^{0}=0.57$. The five different provability distributions $P_{1}, P_{2}, \ldots, P_{5}$ that are functions of $F_{2}$ are summarized in Table VI, where case (a) corresponds to the conventional optimization in which all the fitness is evaluated by FEM, whereas no FE analysis is carried out in case (e). In cases (b), (c) and (d), the probability is set higher as the category label becomes higher. The optimization results are shown in Fig.2. It is found that the results (b)-(e) have almost the same values of $T_{\text {ave }}$ as that in (a), whereas the result (e) has a relatively large torque ripple. The number of FE analysis in (d) is about $10 \%$ of that in (a). Figure 3 shows the numbers of FE analysis per generation in the GA process for the different probabilities. The numbers for $P_{2}, P_{3}$ and $P_{4}$ gradually increase with generation because the individuals classified into good categories tends to increase with generation. It is concluded that use of $\mathrm{CNN}$ can reduce the computing cost to $10 \%$ with little change in the performance of the optimized IPM motor.

Table II Parameters for FE analysis* and GA

\begin{tabular}{cc}
\hline \hline Number of genes & 42 \\
Number of individuals & 800 \\
Number of children & 160 \\
Number of generations & 100 \\
Current phase angle [degree] & 30 \\
Current effective value [A] & 4.2425 \\
Number of turns [turn] & 35 \\
Residual flux density [T] & 1.25 \\
\hline \hline
\end{tabular}

*A customized (in-house) software is used.

Table III Category labels for the data obtained by solving (3)

\begin{tabular}{cccccc}
\hline \hline$T_{\text {ave }}$ & $(0,0.45)$ & $(0.45,0.55)$ & $\ldots$ & $(0.95,1.05)$ & $(1.05, \infty)$ \\
$\hat{T}_{\text {ave }}$ & 0 & 0.5 & $\ldots$ & 1.0 & 1.1 \\
\hline$T_{\text {rip }}$ & $(0,0.65)$ & $(0.65,0.75)$ & $\cdots$ & $(1.15,1.25)$ & $(1.25, \infty)$ \\
$\widehat{T}_{\text {rip }}$ & 0 & 0.7 & $\cdots$ & 1.2 & 1.3 \\
\hline \hline
\end{tabular}

$\overline{\mathrm{T}}_{\text {ave }}$ and $\widehat{T}_{\text {rip }}$ denote the category labels for $T_{\text {ave }}$ and $T_{\text {rip }}$, respectively.

Table IV $T_{\text {ave }}$ evaluated by CNN and FEM

\begin{tabular}{|c|c|c|c|c|c|c|c|c|c|c|}
\hline & \multicolumn{9}{|c|}{ Label by CNN } \\
\hline & & 0 & 0.5 & 0.6 & 0.7 & 0.8 & 0.9 & 1 & 1.1 & TOTAI \\
\hline \multirow{9}{*}{ 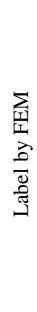 } & 0 & 520 & 29 & 4 & 3 & 2 & 1 & 0 & 0 & 559 \\
\hline & 0.5 & 15 & 452 & 47 & 1 & 0 & 0 & 0 & 0 & 515 \\
\hline & 0.6 & 0 & 38 & 482 & 49 & 0 & 0 & 0 & 0 & 569 \\
\hline & 0.7 & 0 & 1 & 38 & 518 & 23 & 0 & 0 & 0 & 580 \\
\hline & 0.8 & 0 & 0 & 0 & 18 & 390 & 12 & 0 & 0 & 420 \\
\hline & 0.9 & 0 & 0 & 0 & 3 & 18 & 473 & 15 & 0 & 509 \\
\hline & 1 & 0 & 0 & 0 & 0 & 0 & 10 & 396 & 16 & 422 \\
\hline & 1.1 & 0 & 0 & 0 & 0 & 0 & 0 & 8 & 418 & 426 \\
\hline & TOTAL & 535 & 520 & 571 & 592 & 433 & 496 & 419 & 434 & 4000 \\
\hline
\end{tabular}

\section{B. Application to different model}

Here, we consider the application of the trained CNN to different electric-motor model as an example of (ii) in Table I. In the learning phase, $\mathrm{CNN}$ is trained with the data obtained by the topology optimization of motor models 1 and 3 shown in

Table V $T_{\text {rip }}$ evaluated by CNN and FEM

\begin{tabular}{|c|c|c|c|c|c|c|c|c|c|c|}
\hline & \multicolumn{9}{|c|}{ Label by CNN } \\
\hline & & 0.6 & 0.7 & 0.8 & 0.9 & 1 & 1.1 & 1.2 & 1.3 & TOTAL \\
\hline \multirow{9}{*}{ 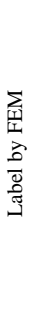 } & 0.6 & 627 & 411 & 53 & 51 & 7 & 28 & 17 & 25 & 1219 \\
\hline & 0.7 & 182 & 644 & 186 & 72 & 12 & 14 & 12 & 23 & 1145 \\
\hline & 0.8 & 50 & 267 & 589 & 187 & 33 & 38 & 23 & 15 & 1202 \\
\hline & 0.9 & 25 & 92 & 223 & 689 & 175 & 96 & 44 & 16 & 1360 \\
\hline & 1 & 12 & 59 & 93 & 259 & 425 & 301 & 72 & 27 & 1248 \\
\hline & 1.1 & 10 & 38 & 62 & 105 & 172 & 680 & 240 & 34 & 1341 \\
\hline & 1.2 & 24 & 51 & 56 & 55 & 154 & 295 & 520 & 103 & 1258 \\
\hline & 1.3 & 18 & 42 & 55 & 58 & 16 & 160 & 291 & 587 & 1227 \\
\hline & TOTAL & 948 & 1604 & 1317 & 1476 & 994 & 1612 & 1219 & 830 & 10000 \\
\hline
\end{tabular}

Table VI Probability distributions for problem (4)

\begin{tabular}{|c|c|c|c|c|c|}
\hline \hline Labels of & \multicolumn{5}{|c|}{ probabilities [\%] } \\
\cline { 2 - 6 }$F_{2}$ & $P_{1}$ & $P_{2}$ & $P_{3}$ & $P_{4}$ & $P_{5}$ \\
\hline 1.3 & 100 & 100 & 100 & 100 & 0 \\
\hline 1.2 & 100 & 100 & 50 & 25 & 0 \\
\hline 1.1 & 100 & 100 & 25 & 13 & 0 \\
\hline 1 & 100 & 50 & 13 & 7 & 0 \\
\hline 0.9 & 100 & 25 & 7 & 4 & 0 \\
\hline 0.8 & 100 & 13 & 4 & 2 & 0 \\
\hline 0.7 & 100 & 7 & 2 & 1 & 0 \\
\hline 0 & 100 & 0 & 0 & 0 & 0 \\
\hline \hline
\end{tabular}

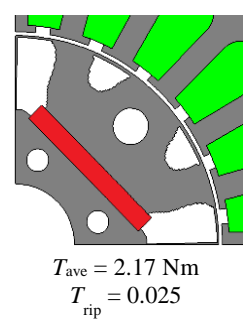

(a) $P_{1}$

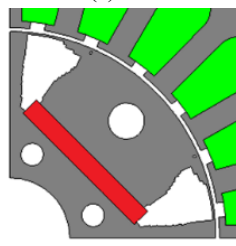

$T_{\text {ave }}=2.29 \mathrm{Nm}$ $T_{\text {rip }}=0.134$

(c) $P_{3}$

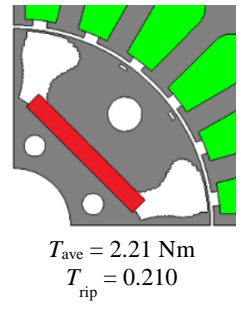

(e) $P_{5}$

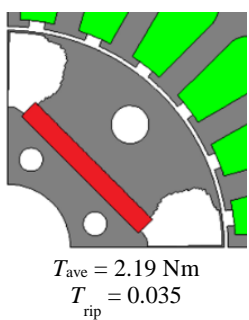

(b) $P_{2}$

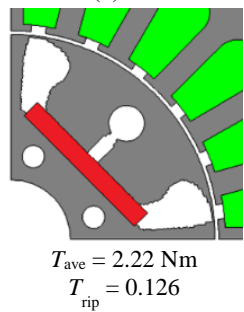

(d) $P_{4}$

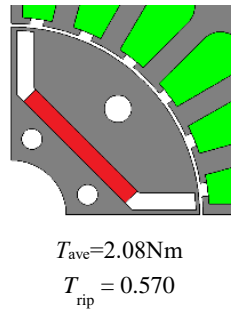

(f) Reference model [9]
Fig.2 Shapes optimized with CNN trained by the different problem. Probability distributions $P_{i}, i=1,2, \ldots, 5$, in Table VI are used to solve problem (4). 
Fig. 4, where problem (3) is solved. The trained $\mathrm{CNN}$ is then used for the optimization of motor model 2 in Fig. 4. The average torque of model 2 is evaluated by the trained CNN. The classification results with respect to $T_{\text {ave }}$ provided by FEM and CNN are summarized in the confusion matrix shown in Table VII. The result seems satisfactory; $96 \%$ samples are within the range of \pm 0.1 . Problem (3) is solved for motor 2 with aid of the trained CNN. The two different probability distributions $P_{1}^{\prime}$ and $P_{2}^{\prime}$ that are functions of $F_{1}$ for execution of $\mathrm{FE}$ analysis are summarized in Table VIII. The resultant shapes obtained with aid of the trained CNN are shown in Fig.5. There are no significant differences both in the rotor shapes and torque performances. The total number of FE analysis in (b) is about $33 \%$ of the that in (a) which corresponds to the topology optimization without DL.

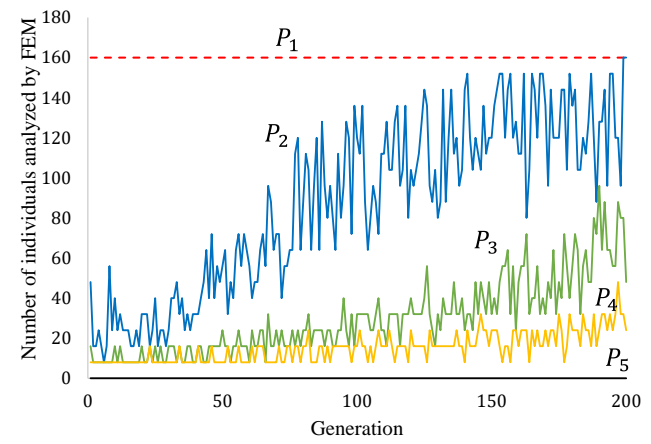

Fig. 3 Change in number of FE analysis

Table VII $T_{\text {ave }}$ evaluated by CNN and FEM for model 2 in Fig.4

\begin{tabular}{|c|c|c|c|c|c|c|c|c|c|c|}
\hline & \multicolumn{9}{|c|}{ Label by CNN } \\
\hline & & 0 & 0.5 & 0.6 & 0.7 & 0.8 & 0.9 & 1 & 1.1 & TOTAL \\
\hline \multirow{9}{*}{ 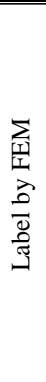 } & 0 & 397 & 76 & 24 & 21 & 16 & 10 & 4 & 0 & 548 \\
\hline & 0.5 & 29 & 360 & 62 & 1 & 0 & 0 & 0 & 0 & 452 \\
\hline & 0.6 & 7 & 83 & 315 & 74 & 1 & 0 & 0 & 0 & 480 \\
\hline & 0.7 & 10 & 1 & 103 & 335 & 76 & 0 & 0 & 0 & 525 \\
\hline & 0.8 & 17 & 0 & 1 & 65 & 286 & 59 & 0 & 0 & 428 \\
\hline & 0.9 & 10 & 0 & 0 & 1 & 96 & 350 & 46 & 0 & 503 \\
\hline & 1 & 0 & 0 & 0 & 0 & 2 & 148 & 374 & 10 & 534 \\
\hline & 1.1 & 0 & 0 & 0 & 0 & 0 & 0 & 269 & 261 & 530 \\
\hline & TOTAL & 470 & 520 & 505 & 497 & 477 & 567 & 693 & 271 & 4000 \\
\hline
\end{tabular}

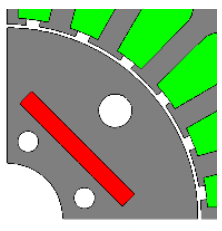

(a) model 1

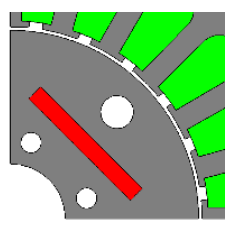

(b) model 2

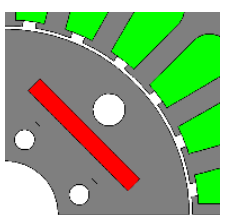

(c) model 3
Fig.4 IPM motors with different position of permanent magnet

Table VIII Probability distributions for problem in III.B

\begin{tabular}{|c|c|c|}
\hline \hline Labels of & \multicolumn{2}{|c|}{ probabilities [\%] } \\
\cline { 2 - 3 }$F_{1}$ & $P_{1}^{\prime}$ & $P_{2}^{\prime}$ \\
\hline 1.1 & 100 & 100 \\
\hline 1.0 & 100 & 25 \\
\hline 0.9 & 100 & 13 \\
\hline 0.8 & 100 & 7 \\
\hline 0.7 & 100 & 4 \\
\hline 0.6 & 100 & 1 \\
\hline 0.5 & 100 & 1 \\
\hline 0 & 100 & 0 \\
\hline \hline
\end{tabular}

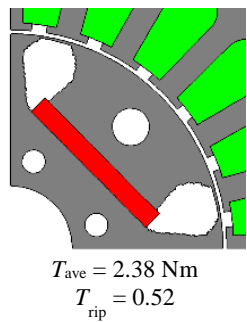

(a) $P_{1}^{\prime}$

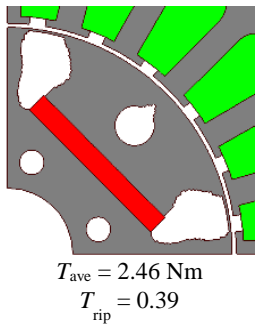

(b) $P_{2}^{\prime}$
Fig.5 Shapes optimized with $\mathrm{CNN}$ trained by different model.

Probability distributions $P_{1}^{\prime}$ and $P_{2}^{\prime}$ in in Table VIII are used to solve problem (3).

\section{CONCLUSIONS}

In this paper, a method based on DL to accelerate the topology optimization using a stochastic optimization method has been proposed. In this method, the individuals in the GA process of the topology optimization are evaluated by the CNN prepared in the learning phase prior to the optimization. The FE analysis for the evaluation of the individuals is performed in accordance with a probability that depends on the categories provided by CNN. It has been shown that the average torque and torque ripple are accurately classified by the trained $\mathrm{CNN}$. The optimization results obtained with aid of the CNN trained for a different problem as well as different models have little differences in the shape and performance compared with those obtained by the conventional method. The computing costs are reduced to about $10 \%$ and $33 \%$, respectively. We plan to develop the method to extract design features necessary for good motor performances from the trained $\mathrm{CNN}$ in future. Moreover we will study the data size and training method to realize the CNN which accurately infers the performances of motors with different magnet shape, location and magnetization as well as different number of poles and teeth.

\section{REFERENCES}

[1] T. Sato, K. Watanabe, H. Igarashi, Multimaterial Topology Optimization of Electric Machines Based on Normalized Gaussian Network, IEEE Trans. Magn., vol. 51, no. 3, 7202604, 2015.

[2] S. Sato, T. Sato, H. Igarashi, Topology Optimization of Synchronous Reluctance Motor Using Normalized Gaussian Network, IEEE Trans. Magn., vol. 51, Issue 3, 8200904, 2015

[3] R. H. Myers, D. C. Montgomery, C. M. Anderson-Cook, Response surface methodology: Process and product optimization using designed experiments, 3rd Ed., Wiley, 2009

[4] L. Lebensztajn, C.A.R. Marretto, M.C. Costa and J.-L. Coulomb, Kriging: a useful tool for electromagnetic device optimization, IEEE Trans. Magn. , vol. 40, no. 2, pp. 1196-1199, 2004

[5] S. Shimokawa, et al., Fast 3-D Optimization of Magnetic Cores for Loss and Volume Reduction, IEEE Trans. Magn., vol. 54, no. 11, 8400904, 2018

[6] Y. LeCun, Y. Bengio, G. Hinton, Deep Learning, Nature, vol.521, 436444, 2015,

[7] H. Sasaki, H. Igarashi, Topology Optimization of IPM Motor with Aid of Deep Learning, presented at ISEM2017, to be published in Int. J. Appl. Electromagnetics and Mech, DOI: 10.3233/JAE-171164.

[8] S. Doi, H. Sasaki, H. Igarashi, Multi-objective Topology Optimization of Rotating Machines Using Deep Learning, presented at CEFC2018, to be published in IEEE Trans. Magn.

[9] Technical report of the institute of electrical engineering of Japan, industry application society, no.776, 2000. 